\title{
Analysis of a Lorenz-Like Chaotic System by Lyapunov Functions
}

\author{
Fuchen Zhang (iD) ${ }^{1,2}$ \\ ${ }^{1}$ Chongqing Key Laboratory of Social Economy and Applied Statistics, College of Mathematics and Statistics, \\ Chongqing Technology and Business University, Chongqing 400067, China \\ ${ }^{2}$ Mathematical Postdoctoral Station, College of Mathematics and Statistics, Southwest University, Chongqing 400716, China \\ Correspondence should be addressed to Fuchen Zhang; zhangfuchen1983@163.com
}

Received 27 March 2019; Revised 17 June 2019; Accepted 1 July 2019; Published 9 July 2019

Academic Editor: Sergey Dashkovskiy

Copyright (c) 2019 Fuchen Zhang. This is an open access article distributed under the Creative Commons Attribution License, which permits unrestricted use, distribution, and reproduction in any medium, provided the original work is properly cited.

\begin{abstract}
In this paper, we investigate the ultimate bound set and positively invariant set of a 3D Lorenz-like chaotic system, which is different from the well-known Lorenz system, Rössler system, Chen system, Lü system, and even Lorenz system family. Furthermore, we investigate the global exponential attractive set of this system via the Lyapunov function method. The rate of the trajectories going from the exterior of the globally exponential attractive set to the interior of the globally exponential attractive set is also obtained for all the positive parameters values $a, b, c$. The innovation of this paper is that our approach to construct the ultimate bounded and globally exponential attractivity sets assumes that the corresponding sets depend on some artificial parameters $(\lambda$ and $m)$; that is, for the fixed parameters of the system, we have a series of sets depending on $\lambda$ and $m$. The results contain the known result as a special case for the fixed $\lambda$ and $m$. The efficiency of the scheme is shown numerically. The theoretical results may find wide applications in chaos control and chaos synchronization.
\end{abstract}

\section{Introduction}

In 1963, Edward Lorenz discovered a chaotic attractor numerically when he studied the Rayleigh-Bénard convection [1]. This discovery stimulated rapid development of chaos theory and a large number of chaotic systems were reported. Since then, chaos phenomenon and chaotic systems have been intensively studied due to great potential applications of chaotic systems in some engineering and technology fields [2-34]. A lot of new chaotic systems can be found, such as Rössler system [2], Chen system [3], Lü system [4], and Lorenz-Stenflo system [12, 23-25]. How to get the bounds of chaotic systems is one of very central problems in the theory of dynamical systems. Bounds for a new chaotic system are very important for the study of the qualitative behavior of a new chaotic system and chaos control. The bounds of the famous Lorenz system have been studied by Leonov et al. in [26-28]. Zhang et al. [17] give the new results of the bounds of the famous Lorenz system and their new results contain the existing results [26-28] as special cases. The bounds of the Lorenz-Stenflo system have been investigated in [23]. How to get the bounds of the Chen system and the Lü system is an important yet nontrivial open problem due to the important potential applications of the Chen system and the Lü system $[29,30]$. Zhang et al. $[16,21,30]$ investigate the open problems of the bounds of the Chen system and the Lü system and get many important results. However, it is very difficult to get the bounds of chaotic systems $[16,21,28]$ and some results have been derived only for some chaotic systems $[16,17,21,28]$. The bounds of a large number of chaotic systems are still unknown. This paper is devoted to computing the bounds of a compact domain, which contains all compact invariant sets of a Lorenz-like chaotic system.

In this paper, we consider the Lorenz-like system [31]:

$$
\begin{aligned}
& \frac{d x}{d t}=-\sigma(x-y)-a y z, \\
& \frac{d y}{d t}=r x-y-x z, \\
& \frac{d z}{d t}=x y-b z,
\end{aligned}
$$




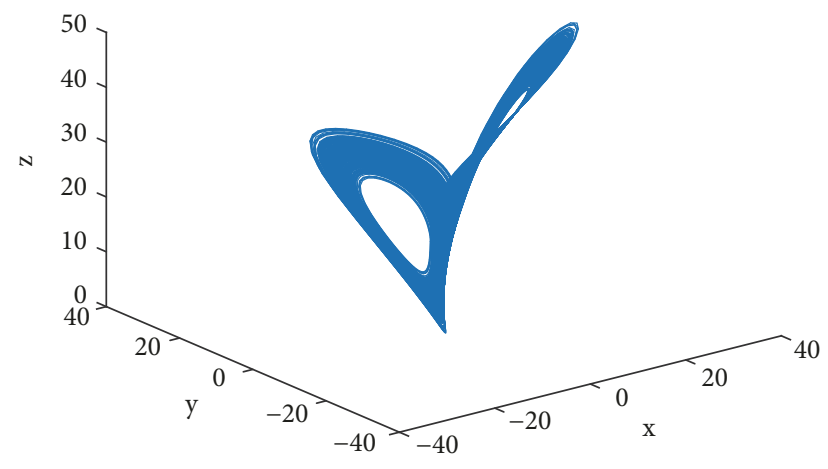

FIGURE 1: Chaotic attractor of system (2) in xOyz space for $a=35$, $b=7$, and $c=25$.

where $r, \sigma, b>0$ and parameter $a$ is real. System (1) is when $a=0$ coincides with the classical Lorenz system [1]; when $\sigma>a r$ and $b=1$, it could be transformed to the Glukhovsky-Dolzhansky system describing fluid convection in the rotating ellipsoidal cavity [32] (see also [8, 15] within this paper), and when $\sigma=-a r$, it could be transformed to the Rabinovich system describing interaction between waves in plasma [33] (see also [8,9] within this paper). When $a=-1$, system (1) coincides with the Qi system [22]:

$$
\begin{aligned}
& \frac{d x}{d t}=a(y-x)+y z, \\
& \frac{d y}{d t}=c x-y-x z, \\
& \frac{d z}{d t}=x y-b z,
\end{aligned}
$$

where $x, y$, and $z$ are real variables; $a, b$, and $c$ are positive parameters of system (2). When $a=35, b=7$, and $c=25$, system (2) is chaotic [22], as shown in Figure 1. System (2) is different from the well-known Lorenz system, Rössler system, Chen system, Lü system, and even Lorenz system family [22]. So, many dynamic behaviors of chaotic system (2) are still unknown, motivating the work to be presented in this paper.

Further in this paper we are going to study the dynamic behavior of system (1) in the special case when $a=-1$, and we compare our results with the results that were obtained previously [31] for the general case $a \in R$.

\section{Dynamic Behavior of System (2)}

Theorem 1. Assume that $\lambda>0, m>0, a>0, b>0$, and $c>0$, with

$$
\begin{aligned}
\Omega_{\lambda, m} & =\left\{(x, y, z) \mid \lambda x^{2}+(\lambda+m) y^{2}\right. \\
+m & {\left.\left[z-\frac{(a+c) \lambda+m c}{m}\right]^{2} \leq R_{\lambda, m}^{2}\right\}, }
\end{aligned}
$$

where

$$
\begin{aligned}
& R_{\lambda, m}^{2} \\
& \quad= \begin{cases}\frac{b^{2}}{4 a(b-a)} \frac{[(a+c) \lambda+m c]^{2}}{m}, & a \geq 1, \quad b \geq 2 a, \\
\frac{b^{2}}{4(b-1)} \frac{[(a+c) \lambda+m c]^{2}}{m}, & a>1, b \geq 2, \\
{[(a+c) \lambda+m c]^{2},} & b<2 a, b<2 .\end{cases}
\end{aligned}
$$

Then, $\Omega_{\lambda, m}$ is the ultimate bound and positively invariant set of system (2).

Proof. Define the Lyapunov-like function

$$
\begin{aligned}
V_{\lambda, m}(X)= & V_{\lambda, m}(x, y, z) \\
= & \lambda x^{2}+(\lambda+m) y^{2} \\
& +m\left[z-\frac{(a+c) \lambda+m c}{m}\right]^{2},
\end{aligned}
$$

$(\forall m>0, \lambda>0)$.

Then, the derivative of $V_{\lambda, m}(X)$ is

$$
\begin{aligned}
& \left.\frac{d V_{\lambda, m}(X)}{d t}\right|_{(2)} \\
& =2 \lambda x \frac{d x}{d t}+2(m+\lambda) y \frac{d y}{d t} \\
& \quad+2 m\left[z-\frac{(a+c) \lambda+m c}{m}\right] \frac{d z}{d t}, \\
& =2 \lambda x(a y-a x+y z)+2(m+\lambda) y(c x-y-x z) \\
& \quad+2 m\left[z-\frac{(a+c) \lambda+m c}{m}\right](x y-b z), \\
& =-2 a \lambda x^{2}-2(m+\lambda) y^{2}-2 m b z^{2} \\
& \quad+2 b[(a+c) \lambda+m c] z .
\end{aligned}
$$

Let $d V_{\lambda, m}(X) / d t=0$, and we can get a bounded closed set $\Gamma$ :

$$
\begin{aligned}
\Gamma= & \left\{(x, y, z) \mid \frac{\lambda x^{2}}{b[(a+c) \lambda+m c]^{2} / 4 a m}\right. \\
& +\frac{(m+\lambda) y^{2}}{b[(a+c) \lambda+m c]^{2} / 4 m} \\
& \left.+\frac{m[z-((a+c) \lambda+m c) / 2 m]^{2}}{[(a+c) \lambda+m c]^{2} / 4 m}=1\right\} .
\end{aligned}
$$

Since chaotic system (2) is bounded, the continuous function (5) can reach its maximum value on the bounded closed set $\Gamma$ above.

Hence, solutions of chaos system (2) are contained in the set defined by $\left\{(x, y, z) \mid V_{\lambda, m}(X) \leq \max V_{\lambda, m}(X)=\right.$ 
$\left.R_{\lambda, m}^{2}, X \in \Gamma\right\}$. We will get the maximum value of function (5) on $\Gamma$ by dealing with the conditional extremum problem below:

$$
\begin{aligned}
\max & V_{\lambda, m}(X) \\
= & \max \left\{\lambda x^{2}+(\lambda+m) y^{2}+m[z\right. \\
- & \frac{\left.(a+c) \lambda+m c]^{2}\right\}}{m}, \\
\text { s.t. } & \frac{\lambda x^{2}}{b[(a+c) \lambda+m c]^{2} / 4 a m} \\
+ & \frac{(m+\lambda) y^{2}}{b[(a+c) \lambda+m c]^{2} / 4 m} \\
+ & \frac{m[z-((a+c) \lambda+m c) / 2 m]^{2}}{[(a+c) \lambda+m c]^{2} / 4 m}=1 .
\end{aligned}
$$

Denote

$$
\begin{aligned}
\sqrt{\lambda} x & =x_{1}, \\
\sqrt{\lambda+m} y & =y_{1}, \\
\sqrt{m} z & =z_{1}, \\
\frac{(a+c) \lambda+m c}{2 \sqrt{m}} & =l, \\
\frac{b[(a+c) \lambda+m c]^{2}}{4 a m} & =n^{2}, \\
\frac{b[(a+c) \lambda+m c]^{2}}{4 m} & =k^{2},
\end{aligned}
$$

and then (8) becomes the following form:

$$
\begin{array}{ll}
\max & V_{m, \lambda}(X)=\max \left\{x_{1}^{2}+y_{1}^{2}+\left(z_{1}-2 l\right)^{2}\right\}, \\
\text { s.t. } & \frac{x_{1}^{2}}{n^{2}}+\frac{y_{1}^{2}}{k^{2}}+\frac{\left(z_{1}-l\right)^{2}}{l^{2}}=1 .
\end{array}
$$

We can solve problem (10) according to the optimization method and get the expression of $R_{\lambda, m}^{2}$ as follows:

$$
\begin{aligned}
& R_{\lambda, m}^{2} \\
& \quad= \begin{cases}\frac{b^{2}}{4 a(b-a)} \frac{[(a+c) \lambda+m c]^{2}}{m}, & a \geq 1, b \geq 2 a, \\
\frac{b^{2}}{4(b-1)} \frac{[(a+c) \lambda+m c]^{2}}{m}, & a>1, b \geq 2, \\
{[(a+c) \lambda+m c]^{2},} & b<2 a, b<2 .\end{cases}
\end{aligned}
$$

The proof is thus completed.

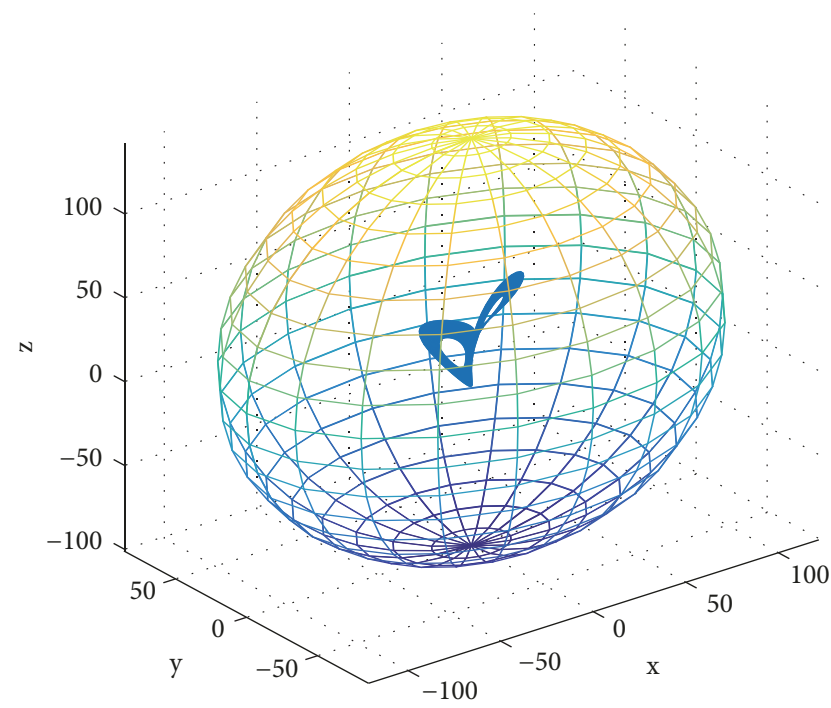

Figure 2: Localization of chaotic attractor of system (2) in xOyz space defined by $\Omega_{1,1}$.

Remark 2. (i) Let us take $m=1$ and $\lambda=1$ in Theorem 1; then we have that

$$
\Omega_{1,1}=\left\{(x, y, z) \mid x^{2}+2 y^{2}+(z-a-2 c)^{2} \leq L^{2}\right\}
$$

is the ultimate bound and positively invariant set of system (2), where

$$
L^{2}= \begin{cases}\frac{b^{2}(a+2 c)^{2}}{4 a(b-a)}, & a \geq 1, b \geq 2 a, \\ \frac{b^{2}(a+2 c)^{2}}{4(b-1)}, & a>1, b \geq 2, \\ (a+2 c)^{2}, & b<2 a, b<2 .\end{cases}
$$

Let us take positive parameters values $a=35, b=7$, and $c=25$ in $\Omega_{1,1}$ above; then we can conclude that

$$
\begin{aligned}
& \Omega_{1,1} \\
& \quad=\left\{(x, y, z) \mid x^{2}+2 y^{2}+(z-20.25)^{2} \leq 121.45^{2}\right\}
\end{aligned}
$$

is the ultimate bound and positively invariant set for system (2). In Figure 2, we show the localization of chaotic attractor of system (2) in xOyz space defined by $\Omega_{1,1}$.

(ii) System (1) in this general form was studied in 1992 by Leonov and Boichenko [31]. Using Lyapunov's direct method, they proved its dissipativity in the sense of Levinson, that is, the existence of a global bounded absorbing set containing global attractor, and also construct several positively invariant sets by stating the following results.

Theorem 3. For an arbitrary solution $(x(t), y(t), z(t))$ of system (1), the following estimate is true:

$$
\lim _{t \rightarrow \infty} \sup \left[y^{2}(t)+(z(t)-r)^{2}\right] \leq l^{2} r^{2},
$$


where

$$
l= \begin{cases}1, & b \leq 2, \\ \frac{b}{2 \sqrt{b-1},} & b>2 .\end{cases}
$$

Theorem 4. Let $2 \sigma-b \geq 0$ and $a(b-2) \geq 0$, and then, for an arbitrary solution $(x(t), y(t), z(t))$ of system (1), the following estimate is true:

$$
\lim _{t \longrightarrow \infty} \inf \left[2(\sigma-a r) z(t)-x^{2}(t)+a y^{2}(t)\right] \geq 0 .
$$

Theorem 5. All trajectories $(x(t), y(t), z(t))$ of system (1) enter the following ellipsoid:

$$
x^{2}+\delta y^{2}+(a+\delta)\left(z-\frac{\sigma+\delta r}{a+\delta}\right)^{2} \leq R^{2},
$$

where

$$
\begin{aligned}
R=\sqrt{\frac{b}{2 c(a+\delta)}}(\sigma+\delta r), & \\
& \delta>-a, c=\min \left(\sigma, 1, \frac{b}{2}\right),
\end{aligned}
$$

and stay in it.

(iii) Our approach to construct the ultimate bounded and globally exponential attractivity sets assumes that the corresponding sets depend on some artificial parameters ( $\lambda$ and $m$ ); that is, for the fixed parameters of system (2), we have a series of sets depending on $\lambda$ and $m$. Let us take $\lambda=1$ and $m=\delta-1(\forall \delta>1)$ in (3); then the set $\Omega_{\lambda, m}$ in (3) coincides with (18).

Though Theorem 1 gives the ultimate bound set of system (2), the global exponential attractive sets of system (2) are still unknown. The global exponential attractive sets of system (2) are described by the following theorem.

Theorem 6. Suppose that $a>0, b>0$, and $c>0$, and let $u s$ denote

$$
\begin{aligned}
X(t)= & (x(t), y(t), z(t)), \\
V_{\lambda, m}(X)= & V_{\lambda, m}(x, y, z) \\
= & \lambda x^{2}+(\lambda+m) y^{2} \\
& +m\left[z-\frac{(a+c) \lambda+m c}{m}\right]^{2}, \\
L_{\lambda, m}^{2}= & \frac{b[(a+c) \lambda+c m]^{2}}{\theta m}, \quad \forall \lambda>0, m>0, \\
\theta & \theta=\min (a, b, 1)>0 .
\end{aligned}
$$

Then the estimate

$$
\begin{aligned}
& {\left[V_{\lambda, m}(X(t))-L_{\lambda, m}^{2}\right]} \\
& \quad \leq\left[V_{\lambda, m}\left(X\left(t_{0}\right)\right)-L_{\lambda, m}^{2}\right] e^{-\theta\left(t-t_{0}\right)}
\end{aligned}
$$

holds for system (2).
Hence, by definition, $\Psi_{\lambda, m}=\left\{X \mid V_{\lambda, m}(X) \leq L_{\lambda, m}^{2}\right\}$ is the globally exponential attractive set of system (2); that is, $\varlimsup_{t \longrightarrow+\infty} V_{\lambda, m}(X(t)) \leq L_{\lambda, m}^{2}$.

Proof. Define

$$
\begin{aligned}
V_{\lambda, m}(X)= & V_{\lambda, m}(x, y, z) \\
= & \lambda x^{2}+(\lambda+m) y^{2} \\
& +m\left[z-\frac{(a+c) \lambda+m c}{m}\right]^{2}, \\
& \forall \lambda>0, m>0,
\end{aligned}
$$

and then the derivative of $V_{\lambda, m}(X)$ is

$$
\begin{aligned}
& \left.\frac{d V_{\lambda, m}(X)}{d t}\right|_{(2)} \\
& =2 \lambda x \frac{d x}{d t}+2(m+\lambda) y \frac{d y}{d t} \\
& +2 m\left[z-\frac{(a+c) \lambda+m c}{m}\right] \frac{d z}{d t} \\
& =2 \lambda x(a y-a x+y z)+2(m+\lambda) y(c x-y-x z) \\
& +2 m\left[z-\frac{(a+c) \lambda+m c}{m}\right](x y-b z), \\
& =-2 a \lambda x^{2}-2(m+\lambda) y^{2}-2 m b z^{2} \\
& +2 b[(a+c) \lambda+m c] z, \\
& \leq-a \lambda x^{2}-(m+\lambda) y^{2}-m b z^{2} \\
& +2 b[(a+c) \lambda+m c] z, \\
& =-a \lambda x^{2}-(m+\lambda) y^{2}-m b\left(z-\frac{(a+c) \lambda+m c}{m}\right)^{2} \\
& +b \frac{[(a+c) \lambda+m c]^{2}}{m} \\
& \leq-\theta V_{\lambda, m}(X)+b \frac{[(a+c) \lambda+m c]^{2}}{m}, \\
& \leq-\theta\left[V_{\lambda, m}(X)-L_{\lambda, m}^{2}\right] .
\end{aligned}
$$

So, we have

$$
\begin{aligned}
& {\left[V_{\lambda, m}(X(t))-L_{\lambda, m}^{2}\right]} \\
& \quad \leq\left[V_{\lambda, m}\left(X\left(t_{0}\right)\right)-L_{\lambda, m}^{2}\right] e^{-\theta\left(t-t_{0}\right)} .
\end{aligned}
$$

And

$$
\varlimsup_{t \longrightarrow+\infty} V_{\lambda, m}(X(t)) \leq L_{\lambda, m}^{2}
$$

By definition, $\Psi_{\lambda, m}=\left\{X \mid V_{\lambda, m}(X) \leq L_{\lambda, m}^{2}\right\}$ is the globally exponential attractive set of system (2).

The proof is thus completed. 


\section{Conclusion}

The article is devoted to study the global behavior of the 3D Lorenz-like chaotic system. For the considered system, we obtain the positive invariant set (ultimate bound) and globally exponential attractive set using Lyapunov function theory and optimization method. Numerical simulations are consistent with the results of theoretical analysis. It is expected that the basic ideas presented in this paper can be applied to explore the bounds of similar chaotic systems in other papers.

\section{Data Availability}

The data used to support the findings of this study are included within the article.

\section{Conflicts of Interest}

The authors declare that there are no conflicts of interest regarding the publication of this paper.

\section{Acknowledgments}

This work was supported by the Scientific and Technological Research Program of Chongqing Municipal Education Commission (Grants nos. KJQN201800818 and KJ1500605), the National Natural Science Foundation of China (Grants nos. 11871122, 11501064, and 11426047), the Basic and Advanced Research Project of CQCSTC (Grant no. cstc2014jcyjA00040), the Research Fund of CTBU (Grant no. 2014-56-11), China Postdoctoral Science Foundation (Grant no. 2016M590850), and Chongqing Postdoctoral Science Foundation Special Funded Project (Grant no. Xm2017174). The author thanks Professors Guanrong Chen in City University of Hong Kong, Gennady A. Leonov in Russian Academy of Sciences, Jinhu Lü in Chinese Academy of Sciences, Xiaofeng Liao in Chongqing University, Qigui Yang in South China University of Technology, Gaoxiang Yang in Ankang University, and Min Xiao in Nanjing University of Posts and Telecommunications for their help.

\section{References}

[1] E. N. Lorenz, "Deterministic non-periodic flow," Journal of the Atmospheric Sciences, vol. 20, pp. 130-141, 1963.

[2] O. E. Rössler, "An equation for continuous chaos," Physics Letters A, vol. 57, no. 5, pp. 397-398, 1976.

[3] G. Chen and T. Ueta, "Yet another chaotic attractor," International Journal of Bifurcation and Chaos, vol. 9, no. 7, pp. 14651466, 1999.

[4] J. Lü and G. Chen, "A new chaotic attractor coined," International Journal of Bifurcation and Chaos, vol. 12, no. 3, pp. 659661, 2002.

[5] E. M. Elsayed, "Expression and behavior of the solutions of some rational recursive sequences," Mathematical Methods in the Applied Sciences, vol. 39, no. 18, pp. 5682-5694, 2016.

[6] N. V. Kuznetsov, T. N. Mokaev, and P. A. Vasilyev, "Numerical justification of Leonov conjecture on LYApunov dimension of
Rossler attractor," Communications in Nonlinear Science and Numerical Simulation, vol. 19, no. 4, pp. 1027-1034, 2014.

[7] N. V. Stankevich, N. V. Kuznetsov, G. A. Leonov, and L. O. Chua, "Scenario of the birth of hidden attractors in the chua circuit," International Journal of Bifurcation and Chaos, vol. 27, no. 12, Article ID 1730038, 2017.

[8] G. Chen, N. V. Kuznetsov, G. A. Leonov, and T. N. Mokaev, "Hidden attractors on one path: glukhovsky-dolzhansky, lorenz, and rabinovich systems," International Journal of Bifurcation and Chaos, vol. 27, no. 08, Article ID 1750115, 2017.

[9] N. V. Kuznetsov, G. A. Leonov, T. N. Mokaev, A. Prasad, and M. D. Shrimali, "Finite-time Lyapunov dimension and hidden attractor of the Rabinovich system," Nonlinear Dynamics, vol. 92, no. 2, pp. 267-285, 2018.

[10] X.-Y. Wang and M.-J. Wang, "Projective synchronization of nonlinear-coupled spatiotemporal chaotic systems," Nonlinear Dynamics, vol. 62, no. 3, pp. 567-571, 2010.

[11] X. Wang and M. Wang, "A hyperchaos generated from Lorenz system," Physica A: Statistical Mechanics and its Applications, vol. 387, no. 14, pp. 3751-3758, 2008.

[12] L. Stenflo, "Generalized Lorenz equations for acoustic-gravity waves in the atmosphere," Physica Scripta, vol. 53, no. 1, pp. 8384, 1996.

[13] N. V. Kuznetsov, G. A. Leonov, M. V. Yuldashev, and R. V. Yuldashev, "Hidden attractors in dynamical models of phaselocked loop circuits: limitations of simulation in MATLAB and SPICE," Communications in Nonlinear Science and Numerical Simulation, vol. 51, pp. 39-49, 2017.

[14] G. A. Leonov, "General existence conditions of homoclinic trajectories in dissipative systems. Lorenz, Shimizu-Morioka, Lu and Chen systems," Physics Letters A, vol. 376, no. 45, pp. 3045-3050, 2012.

[15] G. A. Leonov, N. V. Kuznetsov, and T. N. Mokaev, "Homoclinic orbits, and self-excited and hidden attractors in a Lorenzlike system describing convective fluid motion," The European Physical Journal Special Topics, vol. 224, no. 8, pp. 1421-1458, 2015.

[16] F. Zhang, X. Liao, C. Mu, G. Zhang, and Y. Chen, "On global boundedness of the Chen system," Discrete \& Continuous Dynamical Systems - B, vol. 22, no. 4, pp. 1673-1681, 2017.

[17] F. C. Zhang and G. Y. Zhang, "Further results on ultimate bound on the trajectories of the Lorenz system," Qualitative Theory of Dynamical Systems, vol. 15, no. 1, pp. 221-235, 2016.

[18] F. C. Zhang, X. F. Liao, G. Y. Zhang, and C. L. Mu, "Dynamical analysis of the generalized Lorenz systems," Journal of Dynamical and Control Systems, vol. 23, no. 2, pp. 349-362, 2017.

[19] F. C. Zhang, R. Chen, X. Y. Wang, X. S. Chen, C. L. Mu, and X. F. Liao, "Dynamics of a new 5D hyperchaotic system of lorenz type," International Journal of Bifurcation and Chaos, vol. 28, no. 03, Article ID 1850036, 2018.

[20] F. Zhang, C. Mu, P. Zheng, D. Lin, and G. Zhang, "The dynamical analysis of a new chaotic system and simulation," Mathematical Methods in the Applied Sciences, vol. 37, no. 12, pp. 1838-1846, 2014.

[21] F. Zhang, X. F. Liao, and G. Zhang, "On the global boundedness of the Lü system," Applied Mathematics and Computation, vol. 284, pp. 332-339, 2016.

[22] G. Qi, G. Chen, S. Du, Z. Chen, and Z. Yuan, "Analysis of a new chaotic system," Physica A: Statistical Mechanics and its Applications, vol. 352, no. 2-4, pp. 295-308, 2005. 
[23] P. Wang, D. Li, and Q. Hu, "Bounds of the hyper-chaotic Lorenz-Stenflo system," Communications in Nonlinear Science and Numerical Simulation, vol. 15, no. 9, pp. 2514-2520, 2010.

[24] P. Mukherjee and S. Banerjee, "Projective and hybrid projective synchronization for the Lorenz-Stenflo system with estimation of unknown parameters," Physica Scripta, vol. 82, no. 5, Article ID 055010, 2010.

[25] P. C. Rech, "Spiral organization of periodic structures in the Lorenz-Stenflo system," Physica Scripta, vol. 91, Article ID 075201, 2016.

[26] G. A. Leonov, "Bounds for attractors and the existence of homoclinic orbits in the lorenz system," Journal of Applied Mathematics and Mechanics, vol. 65, no. 1, pp. 19-32, 2001.

[27] G. A. Leonov, A. I. Bunin, and N. Koksch, "Attractor localization of the Lorenz system," Zeitschrift für Angewandte Mathematik und Mechanik, vol. 67, no. 12, pp. 649-656, 1987.

[28] X. Liao, Y. Fu, S. Xie, and P. Yu, "Globally exponentially attractive sets of the family of Lorenz systems," Science China Information Sciences, vol. 51, no. 3, pp. 283-292, 2008.

[29] W.-X. Qin and G. Chen, "On the boundedness of solutions of the Chen system," Journal of Mathematical Analysis and Applications, vol. 329, no. 1, pp. 445-451, 2007.

[30] F. Zhang, C. Mu, and X. Li, "On the boundness of some solutions of the Lü system," International Journal of Bifurcation and Chaos, vol. 22, no. 1, Article ID 1250015, p. 5, 2012.

[31] G. A. Leonov and V. A. Boichenko, "Lyapunov's direct method in the estimation of the Hausdorff dimension of attractors," Acta Applicandae Mathematicae, vol. 26, no. 1, pp. 1-60, 1992.

[32] A. B. Gluhovsky and F. V. Dolzhansky, "Three-component geostrophic models of convection in a rotating fluid," Izvestiya, Atmospheric and Oceanic Physics, vol. 16, pp. 311-318, 1980.

[33] M. I. Rabinovich, "Stochastic self-oscillations and turbulence," Soviet Physics Uspekhi, vol. 21, no. 5, pp. 443-469, 1978.

[34] F. Zhang, C. Mu, S. Zhou, and P. Zheng, "New results of the ultimate bound on the trajectories of the family of the Lorenz systems," Discrete and Continuous Dynamical Systems - Series $B$, vol. 20, no. 4, pp. 1261-1276, 2015. 




Advances in

Operations Research

\section{-n-m}
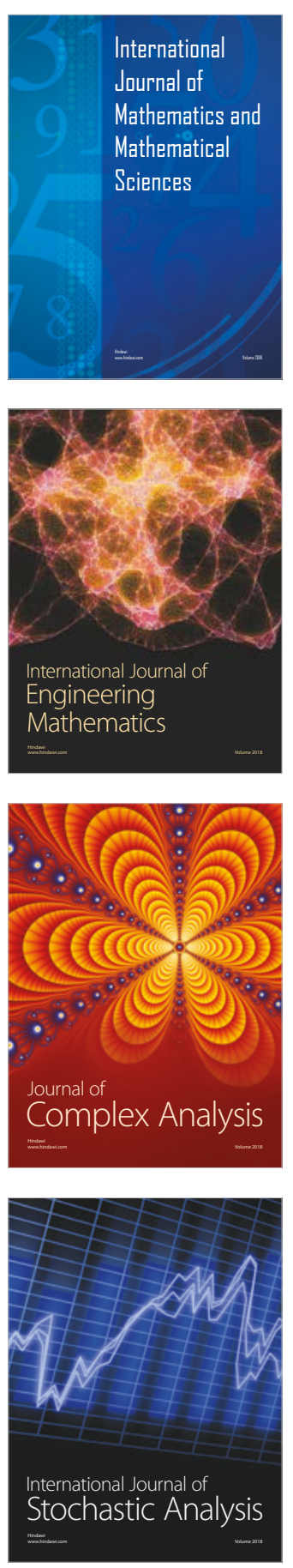
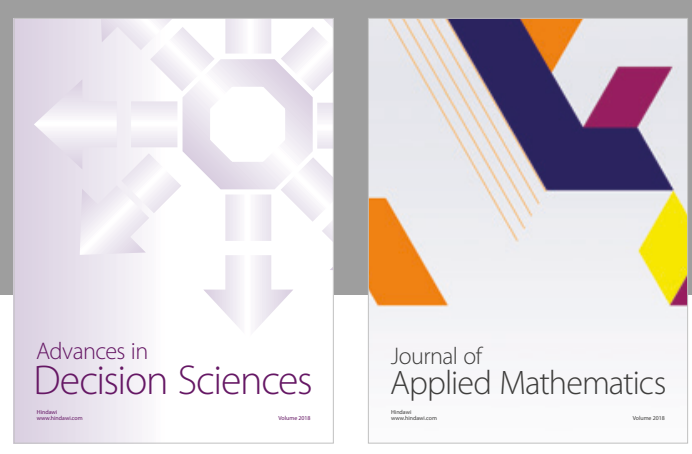

Journal of

Applied Mathematics
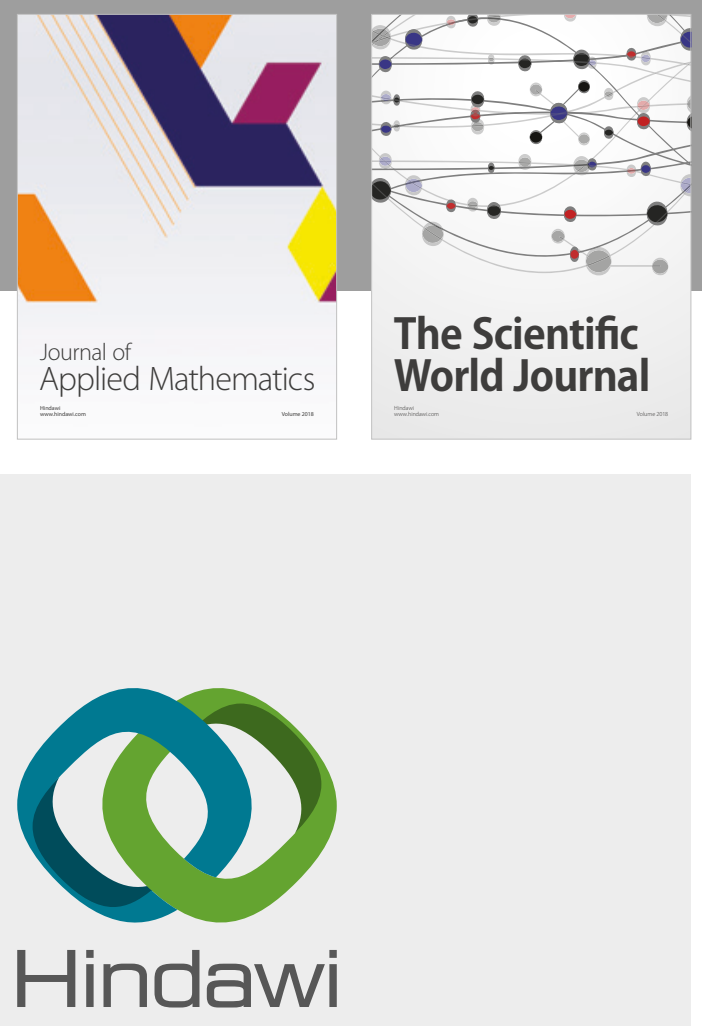

Submit your manuscripts at

www.hindawi.com

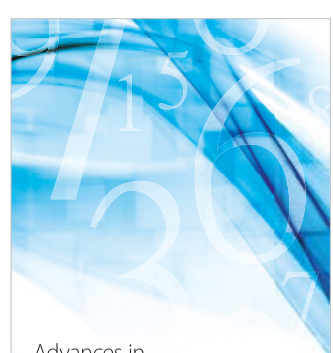

Advances in
Numerical Analysis
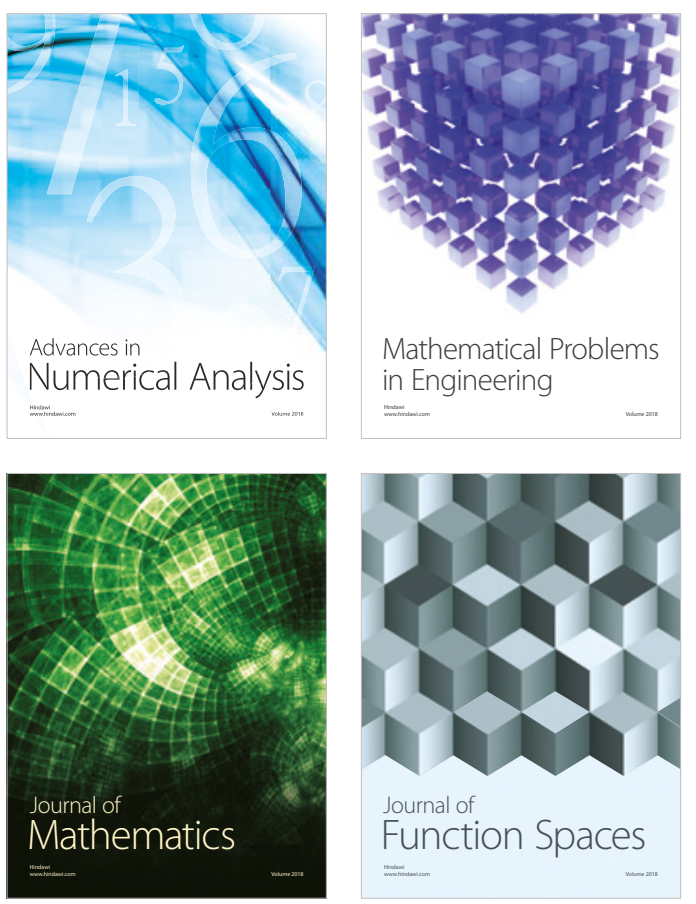

Mathematical Problems in Engineering

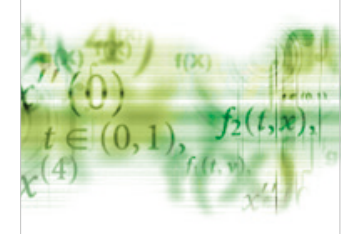

International Journal of

Differential Equations

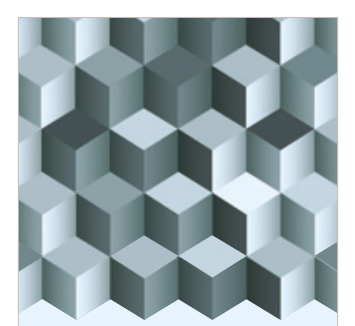

Journal of

Function Spaces
The Scientific

World Journal



Journal of

Probability and Statistics
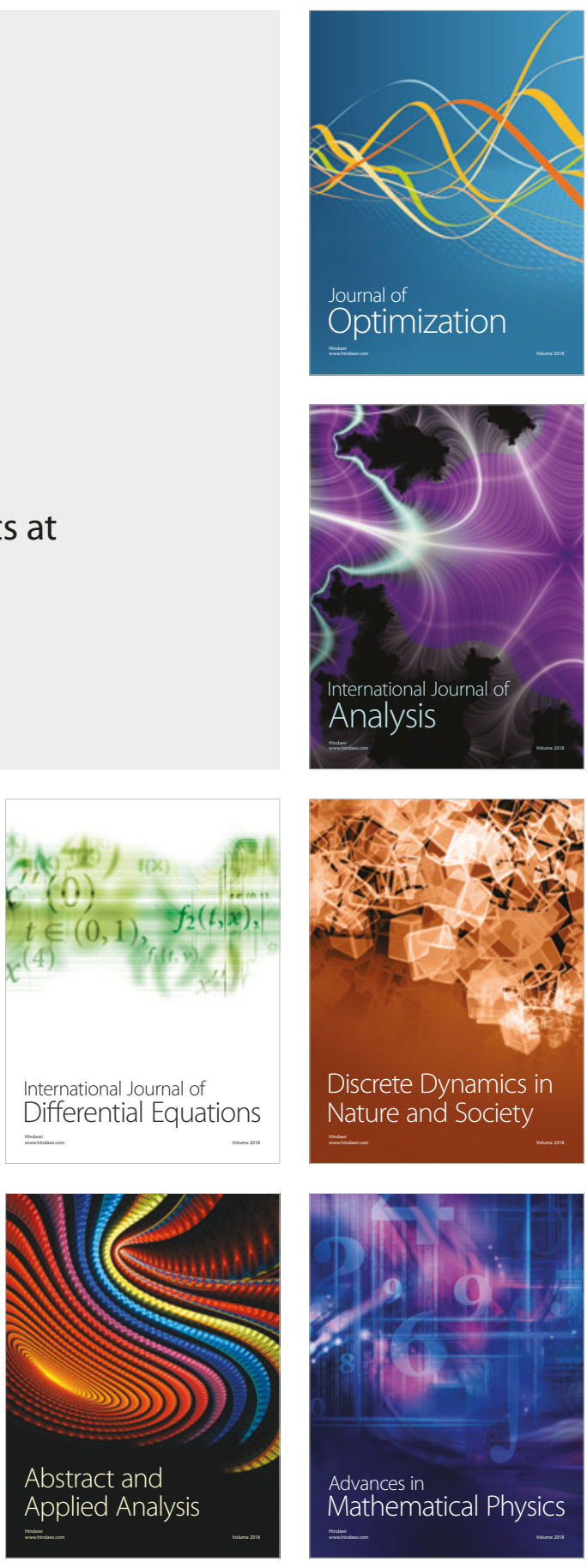